\title{
Quality of life following thoracotomy for lung cancer
}

\author{
Lučka Debevec, Irma Rozman
}

University Clinic of Respiratory and Allergic Diseases Golnik, Golnik, Slovenia

\begin{abstract}
Background. The aim of the study was to assess the preoperative and postoperative quality of life (QoL) in lung cancer patients undergoing thoracotomy and to compare the impairment of QoL in resected and exploratory thoracotomized (ET) patients.

Patients and methods. Forty-three patients age 31 to 82 (mean 61) thoracotomized (lobectomy 29, bilobectomy 1, pneumonectomy 8, ET 5) for non-small cell lung cancer were assessed using the EORTC QLQ-LC30 and QLQ-LC13 questionnaire preoperatively and a mean of $45 \pm 17$ days after the thoracotomy and before eventual chemotherapy and radiation therapy.

Results. After thoracotomy there were significantly impaired functional scales (physical functioning, role functioning, social functioning) and symptom scales (fatigue, constipation, appetite loss, dyspnoea, pain). The remaining symptoms (nausea/vomiting, insomnia, diarrhoea, coughing), global health status, functional scales (emotional functioning, cognitive functioning) and financial difficulties were impaired non-significantly. However, haemoptysis significantly improved and completely disappeared after thoracotomy. There were no significant differences between resected and ET patients.
\end{abstract}

Conclusions. The study established significant impairment of QoL in the first two months after thoracotomy, but no significant differences between resected and ET patients.

Key words: lung neoplasms - surgery; thoracotomy; quality of life

\section{Introduction}

Surgery is the treatment of choice for technical and medical operable non-small cell lung cancer (NSCLC). However, thoracotomy impairs quality of life (QoL) in the case of resection or exploration without resection. Exploratory thoracotomy (ET)

Received 14 August 2007

Accepted 29 August 2007

Correspondence to: Assist. Lučka Debevec, MD, PhD, University Clinic of Respiratory and Allergic Diseases Golnik, SI-4204 Golnik, Slovenia; Phone: +386 42569 100; Fax: +386 42569 117; E-mail: lucka.debevec@ klinika-golnik.si as the only method for tumour verification is currently very rare. In the period 1990 to 1999 the ET rate was 9.1\% among 1808 thoracotomized lung cancer patients at the Department of Thoracic Surgery, Clinical Centre Ljubljana, Slovenia. ${ }^{1}$ Of 131 evaluable ET patients, only one underwent surgery without preoperative verification. ET is mostly caused by unresectability. This was the case in 119/131 patients. Incidental ET (open and closed thoracotomy) could be a consequence of intraoperative complications (1/131 patient) or necessity for pneumonectomy in the case of poor pulmonary function (11/131 patients). In any case, one 
must take into account a certain percent of ET in every large group of thoracotomized lung cancer patients.

During the last ten years, QoL has become an important issue in the treatment of cancer. Especially in clinical trials it is considered an aspect as important or even more important than traditionally used endpoints such as remission rate, diseasefree survival and time to progression. ${ }^{2}$ QoL assessment is a way to obtain objective data in order to "measure" a patient's condition and to evaluate the global impact of therapies administered to improve a patient's situation. $^{3}$ QoL is multidimensional and according to the WHO is a definition of health composed of physical, mental and social function. To measure QoL, the symptoms of a certain tumour are assessed in a semi-quantitative manner. For lung cancer patients the most popular questionnaire is the EORTC (European Organisation for Research and Treatment of Cancer) QLQC30 and its QLQ-LC13 module. ${ }^{4}$ Altogether it has 43 questions that are answered by patients. For converting the answers into percent-values, a special scoring procedure is used $^{5}$ and can be performed in connection with statistical analysis by common computer programs.

The aim of the present study was twofold: (1) to assess preoperative and postoperative QoL in lung cancer patients undergoing thoracotomy and (2) to compare the impairment of QoL in resected and ET patients.

\section{Patients and methods}

From February 2004 to July 2005, 78 patients diagnosed at the University Clinic for Respiratory and Allergic Diseases Golnik, Slovenia, underwent surgery for NSCLC with intention to cure. These patients completed the EORTC QLQ-C30 and QLQLC13 preoperatively. All ET patients (5 of
Table 1. Characteristics of the patients and tumours

\begin{tabular}{ll}
\hline Variable & Value \\
\hline Number of patients & 43 \\
Age (years) - mean & 61 (range 31-82) \\
Male/Female & $33 / 10$ \\
\hline Histology & \\
$\quad$ squamous cell & 21 \\
adenocarcinoma & 16 \\
large cell & 5 \\
non-small cell & 1 \\
\hline
\end{tabular}

Forced expiratory volume in

one second

$81 \pm 20$

$\left(\mathrm{FEV}_{1} \%\right)$-mean

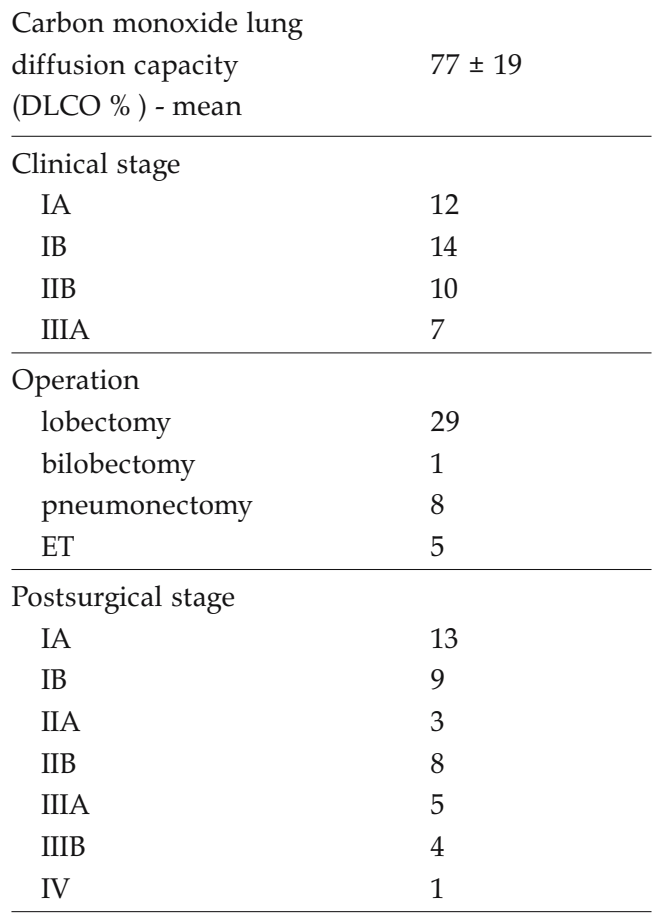

78 patients) and 38 resected patients completed the questionnaire postoperatively again, at $45 \pm 17$ days after thoracotomy, before eventual chemotherapy or radiation therapy. So 43 patients were eligible for the study.

The diagnostic procedure of lung tumour consisted in all patients of chest X-ray, CT 
scan of the chest and upper abdomen, bronchoscopy, pulmonary function testing and arterial blood gas analysis. Cervical mediastinoscopy was performed in 5 patients. Tumour was microscopically confirmed before surgery in all patients.

All patients had the ECOG performance status $\leq 1$. Other characteristics of the patients and tumors are shown in Table 1.

Thirty four patients underwent the anterolateral, 8 axillar, and 1 posterolateral thoracotomy, with a partial rib resection in 12 patients. Pain management postoperatively included epidural analgesia for 2 to 7 (mean 4) days.

The resection was curative without a residue of tumor (R0 stage) in 39 patients, stage R1 in 3, and stage R2 in 1 patient. As postsurgical stage in Table 1 was designated pathological stage in resected patients and surgical stage in ET patients.

The causes of ET were as follows: pleural carcinomatosis in 2, invasion of heart in 1 , extensive invasion of mediastinal lymph nodes in 1, and not permissible pneumonectomy due to poor pulmonary function in 1 patient.

Statistical analysis was carried out using SPSS (Statistical Package for the Social Sciences for Windows, Chicago, IL) version 13.0. The difference was confirmed by paired-samples $t$ test and independent-samples $t$ test respectively. The level of significance was $p<0.05$.

\section{Results}

Preoperative and postoperative QoL is presented in Figure 1 and in Figure 2. Functional scales (physical, role and social functioning) and symptom scales (fatigue, constipation, appetite loss, dyspnoea and pain) significantly worsened (Table 2). In thoracotomized patients it is very important to define the pain. According to presence,

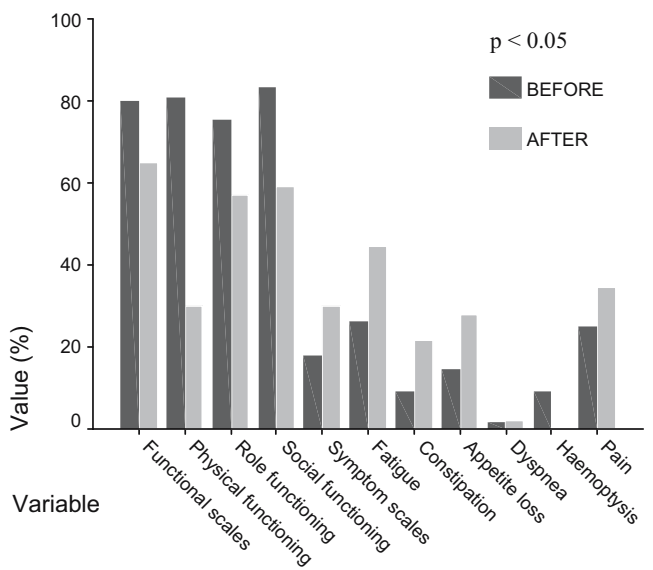

Figure 1. Preoperative and postoperative quality of life in 43 thoracotomized lung cancer patients - significant differences.

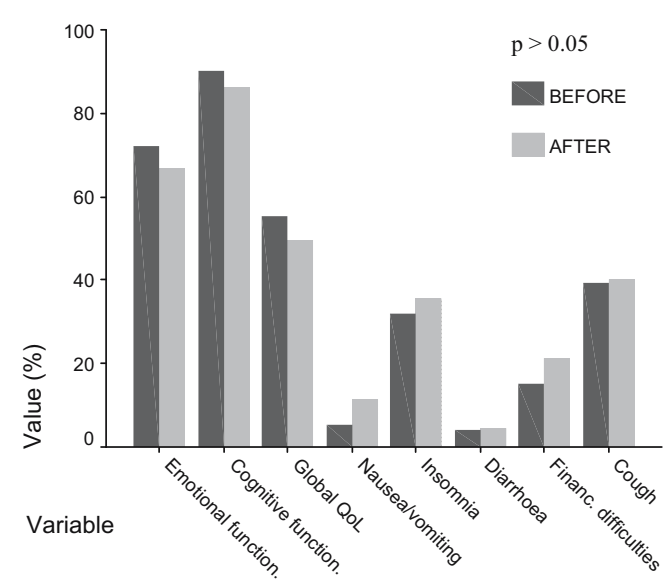

Figure 2. Preoperative and postoperative quality of life in 43 thoracotomized lung cancer patients - non significant differences.

level and location of pain, impairment was significant in unspecified pain $(p=0.030)$, pain interfering with daily activities $(p=$ $0.000)$ and chest pain $(p=0.039)$. There was no significance in the impairment of pain in the arm or shoulder $(p=1.000)$ or of pain in other parts $(p=0.133)$.

QoL was not significantly impaired according to the remaining symptoms (nausea/vomiting, insomnia, diarrhoea, coughing), global health status, functional scales 
Table 2. EORTC QLQ-C30 and QLQ-LC13 scores in 43 patients before and after thoracotomy

\begin{tabular}{|c|c|c|c|c|}
\hline Variables & Item numbers & $\begin{array}{l}\text { Before thoracotomy } \\
\text { Mean } \pm \text { SD }\end{array}$ & $\begin{array}{l}\text { After thoracotomy } \\
\quad \text { Mean } \pm \text { SD }\end{array}$ & p-value \\
\hline Functional scales & $1-7,20-27$ & $80.0 \pm 17.4$ & $64.8 \pm 22.2$ & 0.000 \\
\hline Physical functioning & $1-5$ & $80.9 \pm 20.8$ & $59.8 \pm 33.2$ & 0.000 \\
\hline Role functioning & 6,7 & $75.6 \pm 30.7$ & $57.0 \pm 30.9$ & 0.003 \\
\hline Emotional functioning & $21-24$ & $72.2 \pm 18.8$ & $66,9 \pm 34.0$ & NS \\
\hline Cognitive functioning & 20,25 & $90.3 \pm 16.8$ & $86.0 \pm 18.2$ & NS \\
\hline Social functioning & 26,27 & $83.3 \pm 24.1$ & $59.3 \pm 40.0$ & 0.001 \\
\hline Global QoL & 29,30 & $55.2 \pm 25.6$ & $49.4 \pm 20.7$ & NS \\
\hline Symptom scales/items & $8-19,28$ & $18.1 \pm 15.3$ & $30.0 \pm 16.3$ & 0.000 \\
\hline Fatigue & $10,12,18$ & $26.4 \pm 25.1$ & $44.4 \pm 21.8$ & 0.000 \\
\hline Nausea/vomiting 14, 15 & $5.0 \pm 10.0$ & $11.6 \pm 23.2$ & NS & \\
\hline Insomnia & 11 & $31.8 \pm 35.6$ & $35.7 \pm 29.5$ & NS \\
\hline Constipation & 16 & $9.3 \pm 25.5$ & $21.7 \pm 25.5$ & 0.041 \\
\hline Diarrhoea & 17 & $3.9 \pm 16.6$ & $4,7 \pm 13.8$ & NS \\
\hline Appetite loss & 13 & $14.7 \pm 26.5$ & $27.1 \pm 33.5$ & 0.025 \\
\hline Financial difficulties & 28 & $15.1 \pm 28.7$ & $21.4 \pm 32.8$ & NS \\
\hline Dyspnoea & $8,33-35$ & $1.6 \pm 0.6$ & $1.9 \pm 0.6$ & 0.001 \\
\hline Coughing & 31 & $39.5 \pm 25.5$ & $40.3 \pm 22.5$ & NS \\
\hline Haemoptysis & 32 & $9.3 \pm 16.8$ & $0.0 \pm 0.0$ & 0.001 \\
\hline Pain & $9,19,40-42$ & $24.8 \pm 20.8$ & $34.7 \pm 23.0$ & 0.009 \\
\hline
\end{tabular}

(emotional, cognitive) and financial difficulties. However haemoptysis significantly improved, and completely disappeared after thoracotomy.

In order to compare QoL in resected and ET patients, the differences (impairments or improvements) of single items before and after thoracotomy were computed. Afterwards the resected and ET group were compared according to these QoL differences. Table 3 shows that there were no significant differences between resected and ET patients except in haemoptysis, appearing only preoperatively in resected patients.

\section{Discussion}

Assessing QoL means measuring either the absolute value or relative alteration of quality, and in the case of expressive impairment items may attain a negative value. The goal of the study was the alteration of 
Table 3. The difference of quality of life alteration after thoracotomy in resected and exploratory thoracotomized (ET) patients

\begin{tabular}{|c|c|c|c|c|}
\hline Variables & Item numbers & ET pts Mean \pm SD & Resected pts & p-value Mean \pm SD \\
\hline Functional scales & $1-7,20-27$ & $16.9 \pm 23.0$ & $15.0 \pm 21.7$ & NS \\
\hline Physical functioning & $1-5$ & $36.0 \pm 62.6$ & $19.1 \pm 28.0$ & NS \\
\hline Role functioning & 6,7 & $36.0 \pm 27.4$ & $18.4 \pm 40.2$ & NS \\
\hline Emotional functioning & $21-24$ & $5.0 \pm 19.2$ & $7.7 \pm 33.6$ & NS \\
\hline Cognitive functioning & 20,25 & $-3.3 \pm 7.5$ & $5.3 \pm 19.0$ & NS \\
\hline Social functioning & 26,27 & $10.0 \pm 25.3$ & $25.9 \pm 48.4$ & NS \\
\hline Global QoL & 29,30 & $6.7 \pm 18.0$ & $5.7 \pm 24.2$ & NS \\
\hline Symptom scales/item & $8-19,28$ & $-8.7 \pm 20.0$ & $-12.3 \pm 19.0$ & NS \\
\hline Fatigue & $10,12,18$ & $-8.9 \pm 34.6$ & $-19.3 \pm 29.2$ & NS \\
\hline Nausea/vomiting & 14,15 & $-6.7 \pm 25.3$ & $-6.6 \pm 26.1$ & NS \\
\hline Insomnia & 11 & $-13.3 \pm 29.8$ & $-2.6 \pm 35.8$ & NS \\
\hline Constipation & 16 & $-26.7 \pm 43.5$ & $-10.5 \pm 38.0$ & NS \\
\hline Diarrhoea & 17 & $0.0 \pm 0.0$ & $-0.9 \pm 23.9$ & NS \\
\hline Appetite loss & 13 & $0.0 \pm 40.8$ & $-14.0 \pm 34.3$ & NS \\
\hline Financial difficulties & 28 & $-6.7 \pm 14.9$ & $-6.3 \pm 30.3$ & NS \\
\hline Dyspnoea & $8,33-35$ & $-5.0 \pm 16.2$ & $-11.4 \pm 19.8$ & NS \\
\hline Coughing & 31 & $0.0 \pm 23.6$ & $-0.9 \pm 27.4$ & NS \\
\hline Haemoptysis & 32 & $0.0 \pm 0.0$ & $10.5 \pm 17.5$ & 0.001 \\
\hline Chest pain & 40 & $-13.3 \pm 38.0$ & $-20.2 \pm 62.3$ & NS \\
\hline
\end{tabular}

QoL due to establishing the value of items prior to and 1-2 month after a thoracotomy. Further change of QoL in ET patients is not clearly a consequence of surgery. This might be due to tumor progress or radiation and chemotherapy.

As expected, thoracotomy significantly impaired physical, role, and social functioning, and it increased dyspnoea and chest pain. Any thoracotomy results in a decline in vital capacity of approximately $25 \%$, independent of lung resection, which returns to normal after 6 to 8 weeks. ${ }^{6,7}$ The major effects result from changes in chest wall compliance and an increase in the work of breathing due to the surgical wound and postoperative pain. ${ }^{8}$

It was somewhat surprising that there was no significant change in emotional functioning and financial difficulties. These confirmed both patients' optimism and the good social support in Slovenia. Postoperative constipation could be a consequence of poorer nutrition due to appe- 
tite loss or an effect of some medicines, especially analgesics.

In the literature there are not many articles about the influence of thoracotomy on the QoL in lung cancer patients and we have found no published data on QoL in ET.

Win et al. ${ }^{9}$ assessed the effect of thoracotomy in 110 potentially curatable lung cancer patients using the EORTC QLQ-C30 and QLQ-LC13 before surgery and again at 1, 3 and 6 months postoperatively. Eight ET patients were excluded. Global QoL had deteriorated significantly 1 month after surgery but had returned to preoperative levels by 3 months. Symptoms had worsened significantly at 1 month after surgery but returned to baseline levels by 6 months. Low values of the preoperative QoL were not significantly associated with a poor surgical outcome. However, patients with low preoperative QoL functioning scales and high preoperative symptom scores were more likely to have poor postoperative (6 months) QoL. The only lung function measurement to show a marginally statistically significant association with QoL at 6 months after surgery was the percentage of predicted carbon monoxide transfer factor (DLCO).

Handy et al. ${ }^{10}$ measured QoL in 103 lung cancer patients with the Short-Form 36 Health Survey (SF-36) and Ferrans and Powers Quality-of-Life Index (QLI) preoperatively and 6 months after surgery. Pain and impairment of functional health status persisted for 6 months after resection. DLCO, not forced expiratory volume in one second $\left(\mathrm{FEV}_{1}\right)$, predicted postoperative QoL. Preoperative chemoradiation, the extent of resection, postoperative complications, and adjuvant therapy did not adversely affect functional health status or QoL 6 months after surgery.

Dales et al. ${ }^{11}$ investigated QoL in 91 resected lung cancer patients using the Clinical Dyspnea Index (CDI), Pneumoconiosis
Research Unit Index (PRU), QL-Index (QLI) and Sickness Impact Profile (SIP). QoL was measured preoperatively and 1, 3, 6 and 9 months postoperatively. Dyspnoea significantly increased postoperatively at 1 and 3 months, but returned at 6 and 9 months. Similarly, activities of daily life were significantly impaired at 1 month, and returned to baseline at 6 and 9 months .

Zieren et al. ${ }^{12}$ assessed QoL in 20 lung cancer patients 1 day before surgery, postoperatively on the day of discharge from hospital and at 3-month intervals thereafter until the end of the first postoperative year (6 times) using the EORTC QLQ-C30. The external evaluation was made by a psychologist using the Spitzer Index. After surgery QoL was mainly affected by restrictions related to physical activities, job and household tasks, and disease symptoms, whereas limitations in emotional, social and financial domains were found to be less frequent and less severe. Tumour recurrence was determined to have a significant and negative influence on postoperative QoL. When compared to preoperative assessment, QoL had deteriorated on discharge from hospital but was restored within 3-6 months postoperatively in disease-free patients.

Paull et al. ${ }^{13}$ measured QoL prior to resection, at 0 to 3 months following resection, and at more than 3 months after resection in 37 patients with early-stage NSCLC using Functional Assessment of Cancer Therapy-Lung (FACT-L). Preoperative dyspnoea and postoperative chemotherapy were associated with worse postoperative QoL.

Fiedler et al. ${ }^{14}$ measured QoL in 36 patients 40 months (range 7-147) after pneumonectomy for lung cancer using the EORTC QLQ-C30. Restricted QoL was mainly caused by reduction of lung function due to the loss of parenchyma. Further adjuvant therapy at least 6 months after surgery did not reduce either impairment of lung function or the impairment of QoL. 
Balduyck et al. ${ }^{15}$ assessed QoL in 100 patients undergoing major pulmonary surgery for malignant disease preoperatively and 1, 3, 6 and 12 months postoperatively. Pneumonectomy was signficantly associated with a less favorable QoL score evolution when compared with lobectomy. Comparing antero- and posterolateral thoracotomy, significant differences in pain and dyspnea were seen in favor of the anterolateral technique.

Pompeo et al. ${ }^{16}$ analyzed QoL in 16 patients undergoing tailored combined surgery for stage I lung cancer and severe emphysema using the SF-36 questionnaire. Significant improvements occurred for up 36 months in the general health domain and for 24 months in physical functioning, role physical and general health SF-36 domains. They concluded that selected lung cancer patients with severe emphysema may benefit in terms of long-term QoL.

Myrdal et al. ${ }^{17}$ compared QoL in 112 resected lung cancer patients and 121 patients that underwent coronary bypass surgery using the SF-36 health questionnaire and Hospital Anxiety and Depression Scale (HADS). Lung cancer patients had poorer function because of reduced pulmonary function but showed no sign of increased anxiety or depression. Those that continued to smoke after surgery had impaired mental health.

Li et al. ${ }^{18}$ compared 24 patients resected at thoracotomy and 27 VATS (video-assisted thoracic surgery) resected lung cancer patients 6 months or more after surgery using the EORTC QLQ-C30 and QLQ-LC13. Although VATS patients tended to score higher on the QoL and functioning scales and to report relatively fewer symptoms, there were no significant differences.

Hoang et al. ${ }^{19}$ analyzed the importance of returning to work after thoracic surgery. Return to work is not a trivial component of global post-surgical QoL. Patients have in- dicated that they value being able to return to work as highly as their overall health.

Following the statements above, the greatest impairment of QoL due to thoracotomy was established immediately and in the first 3 months after surgery. QoL improved 6 to 9 months after surgery, but dyspnoea continued in the case of extensive resection and chest pain in some patients. In the case of tumour recurrence or metastatic spread, QoL depends mainly on these. The results of this study agree with the studies cited. The non-significant difference in dyspnoea impairment in pnemonectomized and ET patients was probably due to the small number of patients in each group.

We are aware of shortcomings of the current study. Due to organizing difficulties it was not possible to assess the QoL in all patients at quite the same interval after thoracotomy. The small number of ET patients and the heterogeneity of characteristics in the patients and tumours reduce the reliability of the results. Nevertheless the study is the first essay comparing the QoL following the thoracotomy in ET and resected lung cancer patients.

\section{Conclusion}

This study established a significant impairment of QoL, of functional scales (physical, role and social functioning) and symptom scales (fatigue, constipation, appetite loss, dyspnoea, pain) 1-2 months after thoracotomy, but no significant differences between resected and ET patients.

\section{References}

1. Debevec L, Eržen J, Debeljak A, Crnjac A, Kovač V. Exploratory thoracotomy and its influence on the survival of patients with lung cancer. Wien Klin Wochenschr 2006; 118: 479-84. 
2. Beitz J, Grecco C, Justice R. Quality-of-life and points in cancer clinical trials: the U.S. Food and Drug Administration perspective. J Natl Cancer Inst Monogr 1996; 30: 7-9.

3. Sculier JP, Mancini I, Paesmans M, Klastersky J. Quality of life and supportive care. In: Hansen $\mathrm{HH}$, editor. IASLC Textbook of Lung Cancer. London: Martin Dunitz; 2000. p. 337-48.

4. Aaronson NK, Ahmedzai S, Bergman B, Bullinger M, Cull A, Duez NJ, et al. The European Organisation for Research and Treatment of Cancer QLQ-C30: A quality-of-life instrument for use in international clinical trials in oncology. J Natl Cancer Inst 1993; 85. 365-76.

5. Fayers PM, Aaronson NK, Bjordal K, Groenvold M, Curran D, Bottomley A; on behalf of the EORTC Quality of Life Group. The EORTC QLQ-C30 Scoring Manual. $3^{\text {rd }}$ Edition. Brussels: European Organisation for Research and Treatment of Cancer; 2001.

6. Bollinger CT, Perruchoud AP. Functional evaluation of the lung resection candidate. J Thorac Cardiovasc Surg 1998; 11: 198-212.

7. Ferguson MK, Reeder LB, Mick R. Optimizing selection of patients for major lung resection. J Thorac Cardiovasc Surg 1995; 109: 275-81.

8. Lin J, Martinez FJ, Iannettoni MD. The medical evaluation and management of the lung cancer patient pre-treatment. In: Pass HL, Carbone DP, Johnson DH, Minna JD, Turrisi AT, editors. Lung Cancer. Principles and Practice. Philadelphia: Lippincott Williams Wilkins; 2005. p. 400-25.

9. Win T, Sharples L, Wells FC, Ritchie AJ, Munday $\mathrm{H}$, Laroche CM. Effect of lung cancer surgery on quality of life. Thorax 2005; 60: 234-38.

10. Handy JR, Skokan L, Brooks G, Ott GY. What happens to patients undergoing lung cancer surgery? Outcomes and quality of life before and after surgery. Chest 2002; 122: 21-30.

11. Dales RE, Belanger R, Shamji FM, Leech J, Crepeau A, Sachs HJ. Quality-of-life following thoracotomy for lung cancer. J Clin Epidemiol 1994; 147: 1443-9

12. Zieren HU, Mueller JM, Hamberger U, Pichlmaier H. Quality of life after surgical therapy of bronchogenic carcinoma. Eur J Cardio-thorac Surg 1996; 10: $233-7$.

13. Paull DE, Thomas ML, Meade GE, Updyke GM, Arocho MA, Chin HW, et al. Determinants of quality of life in patients following pulmonary resection for lung cancer. Am J Surg 2006; 192: 565-71.
14. Fiedler R, Neef $H$, Hennig $H$, Rosendahl W, Lautenschlaeger C. Lebensqualitaet nach pneumonektomie wegen bronchialkarzinom. Zentralbl Chir 1997; 122: 327-31.

15. Balduyck B, Hendriks J, Lauwers P, Van Schil P. Quality of life evolution after lung cancer surgery: A prospective study in 100 patients. Lung Cancer 2007; 56: 423-31.

16. Pompeo E, De Dominicis E, Ambrogi V, Mineo D, Elia S, Mineo TC. Quality of life after tailored combined surgery for stage I non-small-cell lung cancer and severe emphysema. Ann Thorac Surg 2003; 76: 1821-7.

17. Myrdal G, Valtysdottir S, Lambe M, Stahle E. Quality of life following lung cancer surgery. Thorax 2003; 58: 194-7.

18. Li WW, Lee TW, Lam SS, Ng CS, Sihoe AD, Wan IY, et al. Quality of life following lung cancer resection. Video-assisted thoracic surgery vs thoracotomy. Chest 2002; 122: 584-9.

19. Hoang CD, Osborne MC, Maddaus MA. Return to work after thoracic surgery: an overlooked outcome measure in quality-of-life studies. Thorac Surg Clin 2004; 14: 409-16. 